\title{
Faradaic current in different mullite materials: single crystal, ceramic and cermets
}

Authors:

Gustavo Mata-Osoro $^{(\mathrm{a})}$, José S. Moya ${ }^{(\mathrm{a})}$, Miguel Morales $^{(\mathrm{b})}$, L. Antonio Díaz ${ }^{(\mathrm{c})}$, Hartmut Schneider $^{(\mathrm{d})}$, Carlos Pecharromán ${ }^{(\mathrm{a}) *}$

(a) Instituto de Ciencia de Materiales de Madrid (CSIC), Madrid, Spain.

(b) LabCaF, Facultade de Física, Universidade de Santiago de Compostela (USC), Santiago de Compostela, Spain.

(c) Centro de Investigación en Nanomateriales y Nanotecnología (CINN), (CSIC),Llanera, Spain

(d) Institut für Kristallographie, Universitätzu Köln, Köln, Germany

Faradaic current measurements have been carried out on three different types of mullite: 2:1 mullite single crystals $(E \perp c), 3: 2$ ceramics and $11 \%$ mullite/Mo

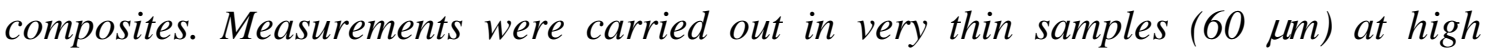
voltages (500 to 1000V). Under these conditions, measurable currents were recorded even at room temperatures. Results indicate notable differences between these three samples, which suggest that, although they share the same name and similar crystalline structure, binding energies and defect distributions seem to be very different. Finally, it has been seen that the excellent behaviour against dielectric breakdown of ceramic mullite does not correspond to those of single crystals or mullite based cermets. 


\section{Introduction.}

From a electromagnetic point of view, mullite is an aluminum-silicate characterized by large insulating and dielectric strength properties[1]. This material is commonly employed in high voltages environments as insulator supporting material and for overvoltage protection[2]. In addition, due to its low thermal expansion and resistance to deformation under load, mullite ceramics is the most common material employed for thermocouple tubing, kiln furniture, and liner material in tube-type furnaces.

Mullite has the general composition $\mathrm{Al}_{4+2 \mathrm{x}} \mathrm{Si}_{2-2 \mathrm{x}} \mathrm{O}_{10-\mathrm{x}}$. According to its formula with $\mathrm{x}-$ values varying between about 0.20 and 0.85 , it contains variable amounts of alumina and silica. Mullite ceramics usually have an alumina/silica ratio of 3:2 (designated as 3:2- or stoichiometric mullite), while single crystals have 2:1 compositions (designated as 2:1-mullite). Depending on the composition, a variable amount of oxygen vacancies is present in mullite, with the $\mathrm{x}$-value of the general formula corresponding to the number of vacancies. Although, 3:2- and 2:1-mullites have similar crystal structures their oxygen defect structure must be different. In the case of 3:2 composition $(x=0.25)$ 1 oxygen vacancy occurs per 4 unit cells, while for 2:1-composition there are 2 oxygen vacancies per 5 unit cells.

It has been suggested that oxygen hopping towards these vacancies may produce some ion conductivity in mullite at high temperature. Although very difficult to measure, some ion conductivity of the ceramic material above $1100^{\circ} \mathrm{C}$ with an activation energy of $4.5 \mathrm{eV}$ has been previously reported [3]. Below this temperature, n-type electronic conduction might be expected to predominate [2]. On doping with transition metallic cations, conductivity increases several orders of magnitude at $400^{\circ} \mathrm{C}[4]$.

Because of the high insulating character of mullite, electric current measurements have not been performed at room temperature yet. In fact, for conductivities of the order of $10^{-12} \mathrm{~S} \cdot \mathrm{m}^{-1}$, only currents below $\mathrm{pA}$ are applied or voltages of $1 \mathrm{~V}$ (a typical large voltage value for impedance measurements) in a regular size sample (10x10x1 mm). Therefore, in order to understand the conduction mechanisms at room temperature, high voltages in addition with very thin samples were applied. For that reason, our experimental setup have optimized for detecting very low currents. Because we have employed a picoamperimeter to detect very low currents, this experimental setup forces 
us to work in the direct current (DC) regime. In this sense, the experimental results cannot be interpreted as usual linear ion conductivity experiments (impedance spectroscopy). In the present case, applied electric fields are large enough to displace charge carriers from shallow potential wells, but not enough to distort the bonding potential of the crystalline structure. It should be noted that under these conditions, several conduction mechanisms can be activated, so that, if the conductivity of all of them are much different, only the less resistive is the one to be detected. In conductive/insulator materials, Schottky and/or tunnel electronic conductivity, can be detected once the voltage exceeds the linear regime $[5,6]$. However, for insulator materials, such as glasses or oxidic ceramics, ion diffusion processes can be activated, in such manner that a faradaic current can be recorded. Because metallic electrodes block the ion current, ion carriers accumulate on the corresponding electrode. Therefore, the dependence of this current with voltage, temperature and time can supply valuable information about electrically charge defects.

\section{Experimental procedure.}

\section{Mullite single crystal growth}

2:1-mullite single crystals with lengths up to $80 \mathrm{~mm}$ and diameters up to $20 \mathrm{~mm}$ were supplied by the Institute of Crystal Growth (Berlin, Germany) using the Czochralski crystal growth technique. The following starting materials were used (wt.\%): $\mathrm{Al}_{2} \mathrm{O}_{3}$ (77.3) and $\mathrm{SiO}_{2}$ (22.7). For a detailed description of the mullite single crystal growth procedure see e.g. Guse and Mateika [7]. Several orientations were cut and polished, but unfortunately, only the piece with $c$-axis parallel to the crystal surface presented the required stability properties to be thinned down to $60 \mu \mathrm{m}$.

\section{Processing of mullite}

Monolithic mullite ceramics were obtained from mullite powders (Scimarek Ltd., Japan) with an average particle size of $1.5 \mu \mathrm{m}$, specific surface area of $7 \mathrm{~m}^{2} / \mathrm{g}$, and chemical analysis (wt.\%) as follows: $\mathrm{Al}_{2} \mathrm{O}_{3}$ (71.5), $\mathrm{SiO} 2(27.3), \mathrm{Na}_{2} \mathrm{O}$ (0.02), $\mathrm{MgO}$ (0.04), $\mathrm{CaO}(0.07)$ and $\mathrm{Fe}_{2} \mathrm{O}_{3}(0.05)$

A suspension of 50 wt.\% solid content (using ethyl alcohol as liquid media) was homogenized by milling with zirconia balls in polyethylene containers at $150 \mathrm{rpm}$ for 18 $\mathrm{h}$ and then dried at $65^{\circ} \mathrm{C}$ for $24 \mathrm{~h}$. The resulting powders were milled in an agate mortar 
and then sieved to a particle size $<43 \mu \mathrm{m}$. Finally, the powders were pressed isostatically at $200 \mathrm{MPa}$, and the resulting compact was sintered in vacuum $\left(5 \times 10^{3} \mathrm{~Pa}\right)$ at $1650^{\circ} \mathrm{C}$ for $1 \mathrm{~h}$, with a heating and cooling rate of $10^{\circ} \mathrm{C} / \mathrm{mm}$.

\section{Processing of mullite/Mo composites}

Monolithic mullite/Mo composites, processing has been previously reported[8]. The following starting materials were used: (1) 99.9\% pure Mo metal (Sigma-Aldrich Co., US) with an average particle size of 1-2 $\mu$ m; (2) Mullite Symulox M72 MC (Nabaltec AG, Germany) with an average particle size of 3-5 $\mu \mathrm{m}$, and with the following chemical composition (wt.\%) $\mathrm{Al}_{2} \mathrm{O}_{3}$ (72.0), $\mathrm{SiO}_{2}$ (26.5), $\mathrm{Na}_{2} \mathrm{O}+\mathrm{K}_{2} \mathrm{O}(0.6), \mathrm{CaO}+\mathrm{MgO}(0.3)$ and $\mathrm{Fe}_{2} \mathrm{O}_{3}(0.3), \mathrm{TiO}_{2}(0.2)$.

Different mullite/Mo composition were mixed in destilled water with 1 wt.\% of deflocculant (Dolapix PC67) addition. All suspensions were homogenized by milling with zirconia balls in polyethylene containers by a Turbula T2F mixer for $24 \mathrm{~h}$.

The slurries used to obtain the composites were dried at $80^{\circ} \mathrm{C}$, for $24 \mathrm{~h}$. The resulting powders were crushed in an agate morter and then passed through a $100 \mu \mathrm{m}$ sieve. Before the sintering process the powders were processed in reduction atmosphere of $\mathrm{H}_{2}$ for 1 hour at $850^{\circ} \mathrm{C}$. The reduced powders were sintered in a Spark Plasma Sintering (SPS) oven at $1500^{\circ} \mathrm{C}$ for 3 minutes in vacuum under $100 \mathrm{MPa}$ pressure.[9]

\section{Electrical measurements}

For electrical measurements, all samples were cut, ground and polished to a thickness of $\sim 0.1 \mathrm{~mm}$. Electrical contacts were made by applying silver paint on the surfaces of the samples by means of circular masks with a fixed area of $15.9 \mathrm{~mm}^{2}$. Current-Voltage(I-V) data were acquired using a Keithley 6517A electrometer as a voltage source and picoampere meter. All measurements were realized inside a faraday cage with a controlled temperature and atmosphere (dry nitrogen) environment. 


\section{Results and Discussion.}

Electric current densities vs. applied field for different temperatures referring to the three mullite materials appear in figures 1 to 3 .

Experimental data have been fitted to the standard model for ion conductivity of ionic conductors[10]

$$
J=2 \lambda q v_{0} n e^{-\frac{W}{k T}} \sinh \left(\frac{q E \lambda}{2 k T}\right)
$$

In this formula, $\lambda, q, v_{0}, n, W$ and $E$ stand for mean free path, carrier charge, jump frequency, carrier volume density, activation energy, and applied electric field respectively. The results of fittings appear in table I. In this table, it has been assumed that $q$ is the electron charge, and $v_{0}$ is fixed to $v_{0}=10^{13} \mathrm{~s}^{-1}$ which is the typical frequency for phonon vibrations, so that, $\lambda$ and $n$ can be estimated, although strong correlation between these two parameters may appear.

In fig. 1 the fitted experimental data corresponding to 2:1-mullite single crystal are shown. For this material, the carrier density is anomalous high while the mean free path very small. However it should be noted that according to the conductivity model (Equation (1)) the mean free path appear multiplying to the internal electric field. In this particular sample, it seems that a leakage current of about $10^{-6} \mathrm{Am}^{-2}$ is present in all the experiment on single crystal. It is not clear the origin of this current, however it seems to be independent of the applied field. Actually, to this current a potential drop should be associated so that it is likely that the microscopic electric field could be smaller than the externally applied. In any case, the fitted activation energy is $0.82 \mathrm{eV}$, which does not depend on the electric field intensity, is similar to the one obtained for oxygen vacancies ion conductors.

Additionally, in fig. 1 can be seen that at fields larger than $10^{7} \mathrm{~V} / \mathrm{m}$ pre-breakdown or electronic conductivity start to appear which suggests that this sample is very fragile and does not support high electric fields. This is contradictory to the common knowledge that states that dielectric strength of mullite is quite high.

In fig.2, the current density plot for a 3:2 mullite ceramic appears For the 3:2-mullite ceramic sample, the current density is notably smaller than in the case of 2:1 single crystal. Although this fact is in agreement with all the previous knowledge about the insulating properties of mullite, it introduces a new question about the resemblances 
between mullite single crystal and ceramics. The mean free path is much larger and the carrier density much smaller than those corresponding to the 2:1 mullite single crystal (Table I). However, the activation energy is smaller $(0.7 \mathrm{eV})$ than in the case of the single crystal.

The last considered system is a ceramic/metal composite with a metal concentration below the percolation threshold (11 vol. \%). It is expected that this composite will present different values of conductivity and activation energy, due to the effect of the metal and some possible oxide which can diffuse along the mullite crystalline structure. In fig.3, the conductivity values for this composite can be seen.

The fitted values for this sample (Table I) indicate that the carrier density in the cermet is slightly higher than in the case of monolithic ceramic, while the mean free path is larger than in the previous case. In addition, the activation energy displays a considerable reduction $(W=0.54 \mathrm{eV})$, which could be justified by the effect of Molybdenum particles which may introduce defects as $\mathrm{MoO}$ or $\mathrm{MoO}_{2}$ due to a partial oxidation of its surface[11].

From the comparison of these three samples several interesting facts can be deduced. In the first place, the mullite single crystal presents conductivity parameters much different from those of ceramics. This odd result can be justified because the chemical composition of both samples is different which suggests that if conductivity were due to oxygen hoppings versus oxygen vacancies (a plausible hypothesis), activation energy and defect density are much different from single crystal to ceramics.

Another important result deduced from conductivity measurements on single crystal is the small dielectric strength of the material. In fact, the sample suffered pre-dielectric breakdown processes at the largest employed fields. This behaviour further indicates that 2:1-mullite has a large concentration of mobile defects and vacancies which can be drifted by the effect of moderate electric field, so that once the faradaic current displaces them to precipitate on the corresponding electrode, crystalline structure becomes unstable and electrochemical breakdown processes [2] take place.

The 3:2-mullite ceramic, presents very good insulating properties. However, even for this system, an ionic current with activation energy of $0.7 \mathrm{eV}$ can be detected. The ionic character of the faradaic current is determined because the resistance of the sample increases with time, as a consequence of depletion of carriers. It should be noted that long term current measurements under high fields have been done on these kinds of 
samples (data not shown) and we have not observed any notable instability apart from a loss of conductivity.

The effects of cations on mullite conductivity has been previously mentioned[4]. In the present case, and due to the processing conditions of mullite/Mo composites, molybdenum oxide appears on the surface of metallic particles. Consequently, after sintering, some molybdenum cations diffuse into the ceramic phase and Mo-doping of mullite is obtained. The energy of activation is reduced to $0.54 \mathrm{eV}$, the carrier density increases in a factor of 2.5 and the mean free path increases more than an order of magnitude. Additionally, this material presents a very poor dielectric strength. Conversely, to the case of mullite ceramics, this materials presents a prominent ageing under high voltages, and as in the case of a single crystal, pre-breakdown and even breakdown processes appear after several days of measurements at constant high field. As it happens also in the case of the single crystal, the amount of defects in the composite is larger than the pure ceramic sample so that it points out as the possible origin of electrical instability. Finally, it should be noted that as it has been shown for $\mathrm{BaTiO}_{3} / \mathrm{Ni}$ composites, which behaves in a similar way as the Mullite/Molybdenum [6, $11,12]$ ones, the electric field can be increased even by a factor of $10^{4}$ by the effect of metallic inclusions, especially for concentrations very close to the percolation threshold. Therefore, the internal electric field could be even one order of magnitude larger than the applied one, so that the very large mean free path value obtained from fitting for the $\mathrm{Mu} / \mathrm{Mo}$ composites could be actually similar to the one corresponding to 3:2 ceramic mullite.

\section{Conclusions.}

As a conclusion, it can be stated that faradaic current is a valuable tool for determining the conductivity mechanisms of highly insulating materials, even at room temperature. The most striking result is that conductivity nature of 2:1 mullite single crystals is very different than 3:2 ceramics. Two possible reasons may account for this:

- A higher carrier density in 2:1 single crystal mullite than in 3:2-mullite ceramics, due to the presence of a higher number of oxygen vacancies enabling higher oxygen ion conductivity.

- The influence of existing (3:2- mullite ceramic) and non-existing (2:1-mullite single crystal) grain boundaries. Obviously this does not have a big influence, 
since the grain boundaries should provide a higher carrier density and thus should increase the conductivity in the ceramic, which has not been observed.

A conclusion is that the number of oxygen vancancies, being higher in 2:1-mullite single crystal and in mullite/Mo composites than in 3:2-mullite ceramics may control the different conductivities of phases. This suggests that the energetic configuration of crystal structures present notable results. In fact, although 3:2-mullite ceramics are well known for their high dielectric strength, both 2:1-mullite single crystals and mullite/Molybdenum composites present poor behaviour against dielectric breakdown. 


\section{References}

1. Schneider, H. and S. Komarneni, Mullite. 2005: Wiley-VCH.

2. Buchanan, R.C., Ceramic materials for electronics. 2004: Marcel Dekker.

3. Rommerskirchen, I., F. Chávez, and D. Janke, Ionic conduction behaviour of mullite (3Al2O3.2SiO2) at 1400 to $1600^{\circ} \mathrm{C}$. Solid State Ionics, 1994. 74(3-4): p. 179-187.

4. Chaudhuri, S.P., S.K. Patra, and A.K. Chakraborty, Electrical resistivity of transition metal ion doped Mullite. Journal of the European Ceramic Society, 1999. 19(16): p. 2941-2950.

5. Jiménez, R., et al. Dielectric and ferroelectric properties of BaTiO3/Ni cermets under high electric fields. in Ferroelectrics. 2002.

6. Pecharromán, C., F. Esteban-Betegón, and R. Jiménez, Electric field enhancement and conduction mechanisms in Ni/BaTiO3 percolative composites. Ferroelectrics, 2010. 400(1): p. 81-88.

7. Guse, W. and D. Mateika, Growth of mullite single-crystals (2A12O3.SIO2) by Czochralski method. Journal of Crystal Growth, 1974. 22(3): p. 237-240.

8. Tomsia, A.P., et al., Powder processing of mullite/Mo functionally graded materials. Journal of the European Ceramic Society, 1998. 18(9): p. 1365-1371.

9. Sivakumar, R., et al., Mullite-molybdenum composites fabricated by pulse electric current sintering technique. Journal of the European Ceramic Society, 2002. 22(5): p. 761-768.

10. Hyde, J.M. and M. Tomozawa, The Relationship Between The DielectricRelaxation And The High-Field Conduction Of Glasses. Physics and Chemistry of Glasses, 1986. 27(4): p. 147-151.

11. Pecharroman, C. and J.S. Moya, Experimental evidence of a giant capacitance in insulator-conductor composites at the percolation threshold. Advanced Materials, 2000. 12(4): p. 294-297.

12. Pecharroman, C., et al., New percolative BaTiO3-Ni composites with a high and frequency-independent dielectric constant (epsilon( $r$ ) approximate to 80,000). Advanced Materials, 2001. 13(20): p. 1541. 
FIGURE CAPTIONS

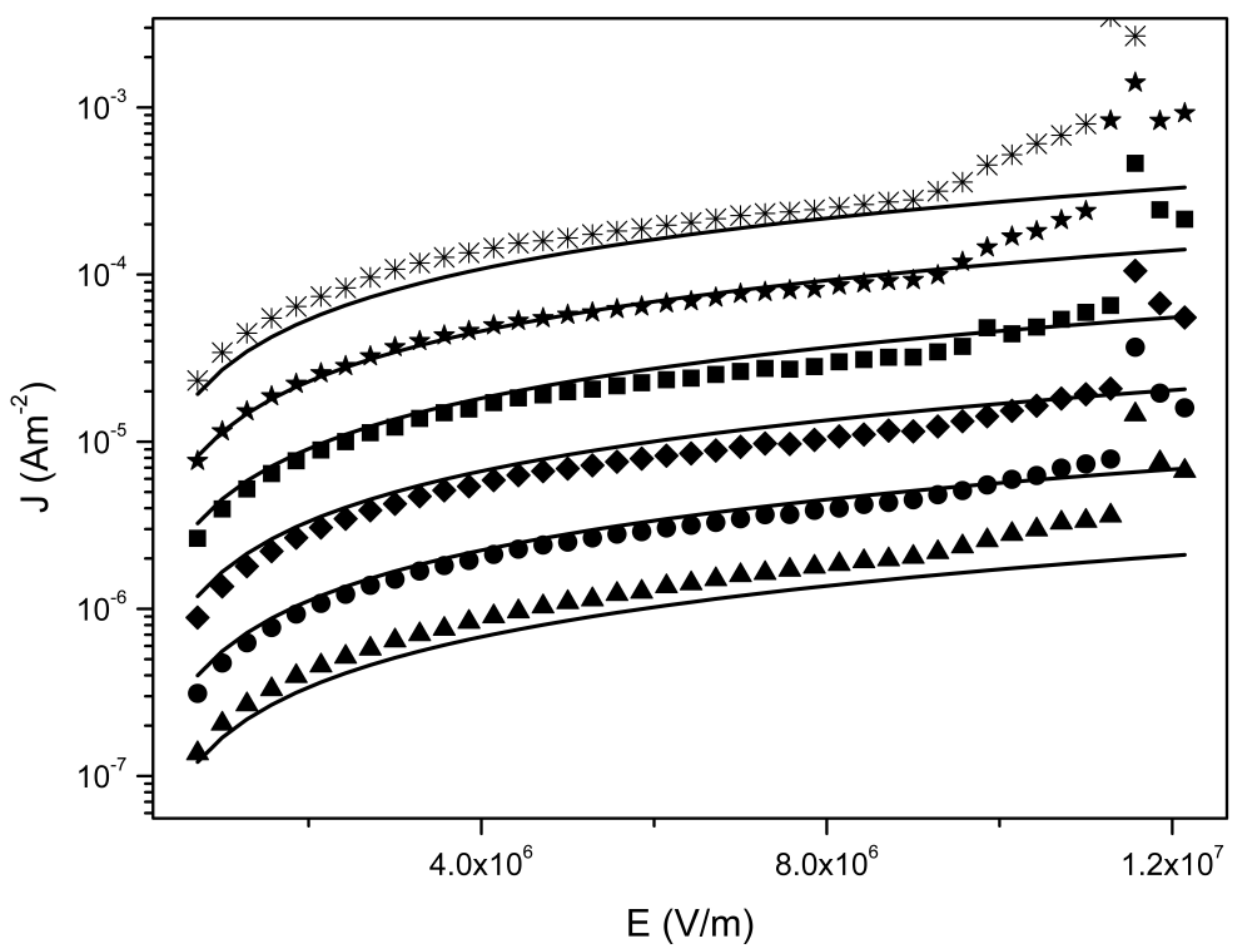

Figure 1. Current density vs Electric field of Mullite single crystal at different temperatures: $\quad(*) 408 \mathrm{~K}(\star) 393 \mathrm{~K}(\bullet) 378 \mathrm{~K}(\bullet) 363 \mathrm{~K}(\bullet) 348 \mathrm{~K}(\bullet) 333 \mathrm{~K}$. Solid lines correspond to calculated data, figures correspond to experimental data. 


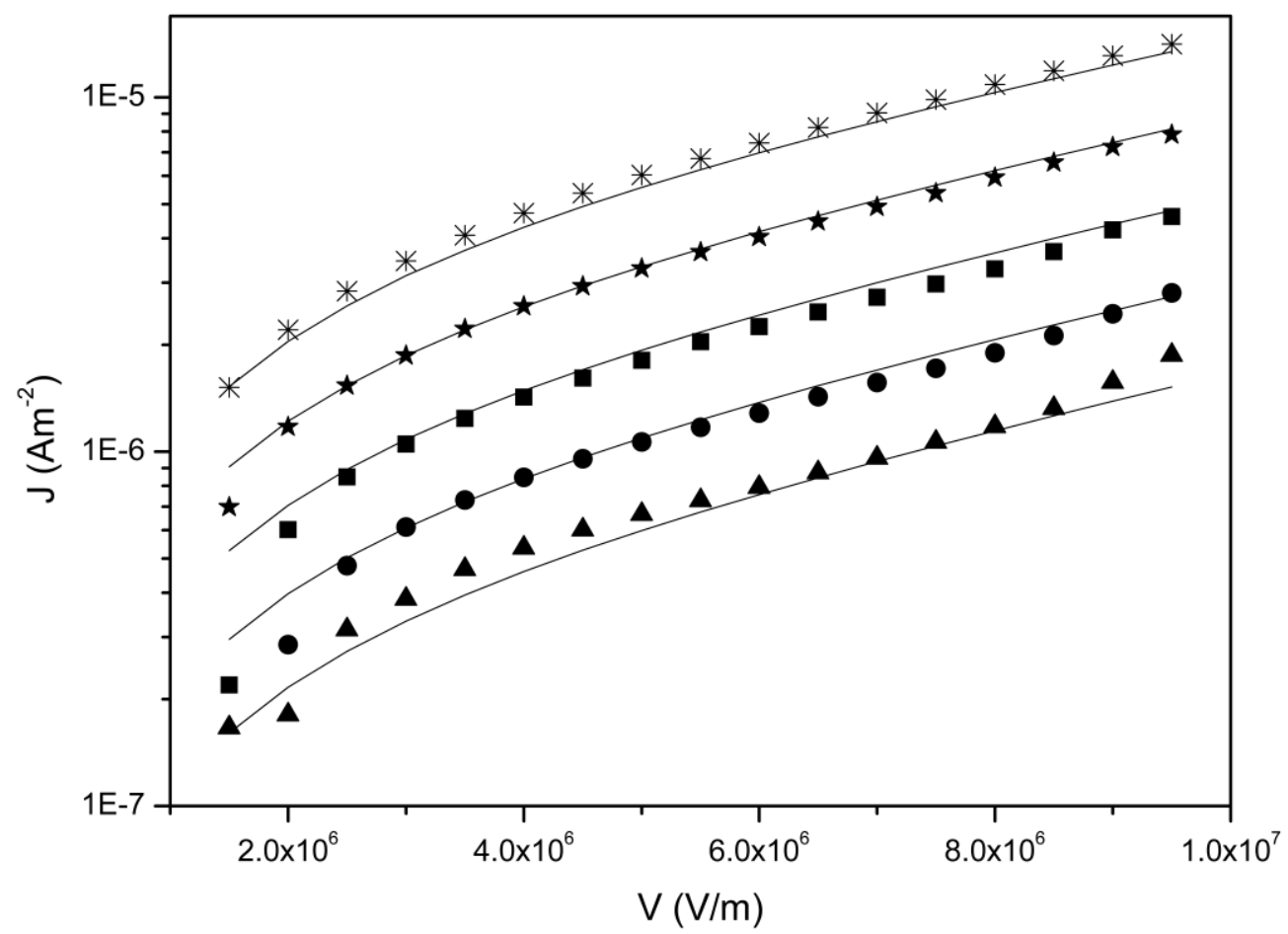

Figure 2. Current density vs Electric field of 3:2 mullite ceramic at different temperatures: $(*) 413 \mathrm{~K}(\star) 403 \mathrm{~K}(\bullet) 393 \mathrm{~K}(\bullet) 383 \mathrm{~K}(\boldsymbol{\bullet}) 373 \mathrm{~K}$.Solid lines correspond to calculated data, figures correspond to experimental data. 


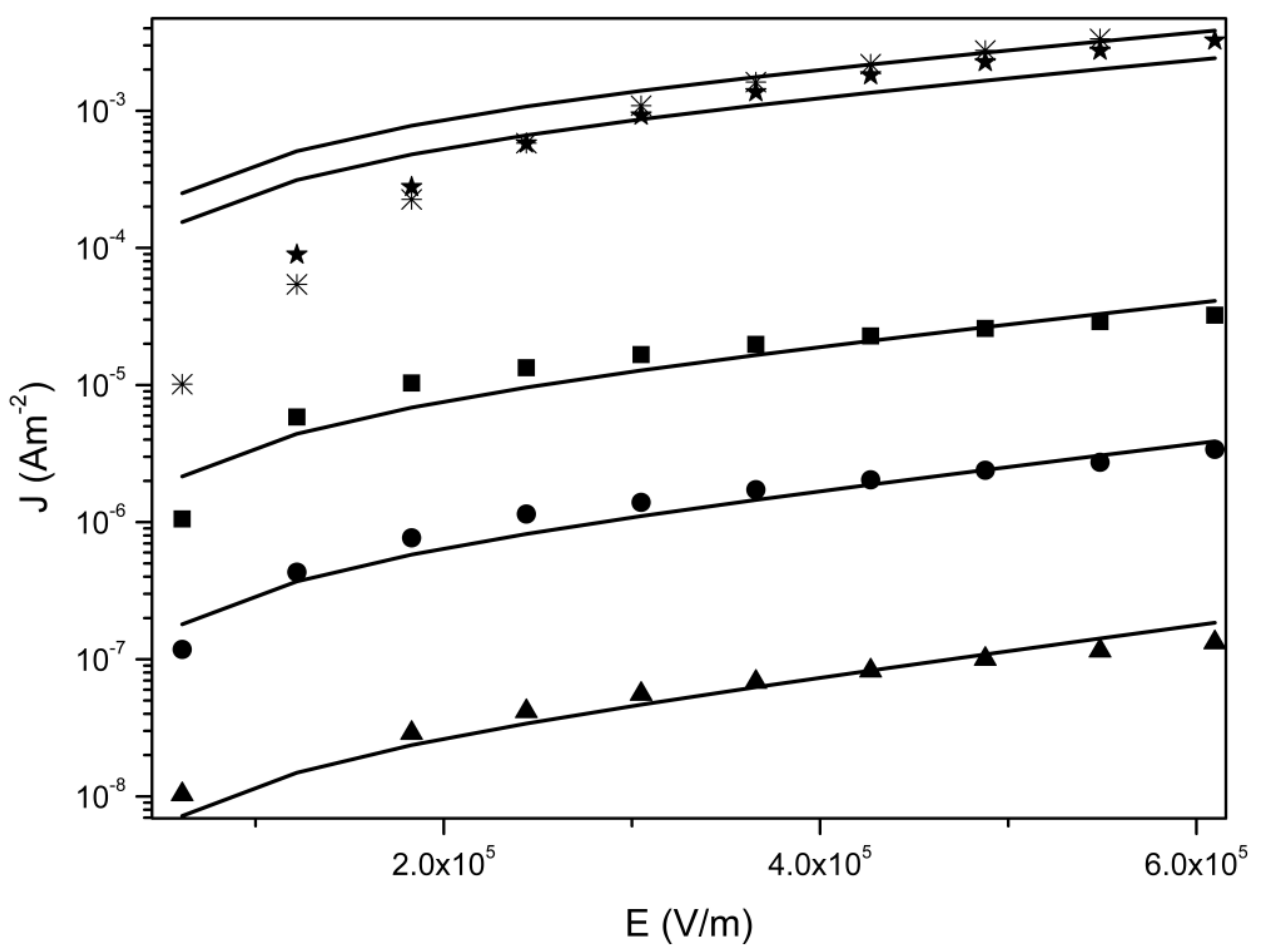

Figure 3. Current density vs Electric field of 3:2 mullite/Mo composite at different temperatures: $(*) 318 \mathrm{~K}(\star) 310 \mathrm{~K}(\bullet) 254 \mathrm{~K}(\bullet) 230 \mathrm{~K}(\boldsymbol{\Delta}) 205 \mathrm{~K}$. Solid lines correspond to calculated data, figures correspond to experimental data.

\section{Table I}

2:1 Mullite SC 3:2 Mullite Cer. 3:2 Mullite/Mo

\begin{tabular}{cccc}
\hline$\lambda(\mathrm{nm})$ & 1.7 & 11 & 151 \\
$n\left(\mathrm{at} / \mathrm{m}^{3}\right)$ & $2.90 \cdot 10^{21}$ & $4.90 \cdot 10^{17}$ & $1.20 \cdot 10^{18}$ \\
$W(\mathrm{eV})$ & 0.82 & 0.7 & 0.54 \\
$E_{\max }(\mathrm{V} / \mathrm{m})$ & $1.00 \cdot 10^{7}$ & $1.00 \cdot 10^{7}$ & $5.00 \cdot 10^{6}$
\end{tabular}

Table 1. Fitted values for experimental data corresponding to the three mullite samples. 